\title{
Comparison between Preoperative Case Optical Coherence Tomography Findings of Carotid Artery Stenosis and Pathological Specimen of Removed Carotid Plaque: Case Report
}

Hiroyuki Matsumoto, ${ }^{1}$ Hideki Takemoto, ${ }^{1}$ Hirokazu Nishiyama, ${ }^{1}$ Yoshiaki Tetsuo, ${ }^{1}$ and Naoyuki Nakao ${ }^{2}$

Objective: Optical coherence tomography (OCT) has recently been applied for the characterization of carotid plaques, but few studies have compared OCT findings with findings from histopathologic examination of carotid plaques. We consider the accuracy, problems, and limitation of OCT findings by comparing OCT findings of carotid artery stenosis and pathological specimen of removed carotid plaque, and confirm that the interpretation of coronary artery findings of OCT can be applied to OCT findings of carotid artery plaque.

Case Presentation: A 63-year-old man with asymptomatic left carotid stenosis underwent carotid endarterectomy (CEA). Findings for histopathologic assessment of excised plaque were compared with those from preoperative OCT images at the same section levels. Preoperative OCT findings of carotid artery stenosis were similar to histopathologic examination of carotid plaques removed by CEA. This suggests that OCT has great ability to visualize pathological fibrous cap and ulceration and to visualize the structure of neovascularization in the neointima. However, OCT was unable to differentiate between lesions that were visualized in histopathological examination as lipid component, hemorrhagic transformation, and necrotic transformation. Conclusion: The high resolution of OCT enables real-time acquisition of carotid plaque data. Although OCT is invasive examination, it may contribute to the evaluation of carotid plaque characterization.

Keywords > optical coherence tomography, carotid artery, carotid plaque, carotid endarterectomy

\section{Introduction}

Optical coherence tomography (OCT) can characterize the microstructure of vascular walls in real time using near infrared rays and a light interferometer. OCT has a 10 -fold higher image resolution than conventional intravascular ultrasound (IVUS) and is able to provide superior intraluminal

${ }^{1}$ Department of Neurological Surgery, Kishiwada Tokushukai Hospital, Kishiwada, Osaka, Japan

${ }^{2}$ Department of Neurological Surgery, Wakayama Medical University, Wakayama, Wakayama, Japan

Received: September 2, 2016; Accepted: December 26, 2016 Corresponding author: Hiroyuki Matsumoto. Department of Neurological Surgery, Kishiwada Tokushukai Hospital, 4-27-1 Kamoricho, Kishiwada, Osaka 596-8522, Japan Email: hiroyuki.matsumoto@tokushukai.jp

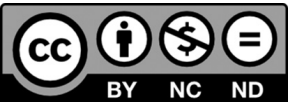

This work is licensed under a Creative Commons Attribution-NonCommercialNoDerivatives International License.

C2017 The Japanese Society for Neuroendovascular Therapy image quality. OCT has been used to characterize plaque in coronary intervention, and it has recently been applied for the characterization of carotid plaque. ${ }^{1-11)}$ However, few studies have compared OCT findings with findings from histopathologic examination of carotid plaques.

Therefore, preoperative OCT findings of carotid artery stenosis were compared with those from histopathologic examination of carotid plaques removed by carotid endarterectomy (CEA). Furthermore, based on this case report, we consider the accuracy, problems, and limitation of OCT findings.

Because OCT imaging is approved only for coronary arteries in Japan, the application to carotid arteries was previously approved by our institutional ethics committee. In advance, we also carefully informed consent to the patient about the risks of the OCT examination.

\section{Case Presentation}

A 63-year-old man developed posterior circulation minor stroke with vertigo and was incidentally noted to have 

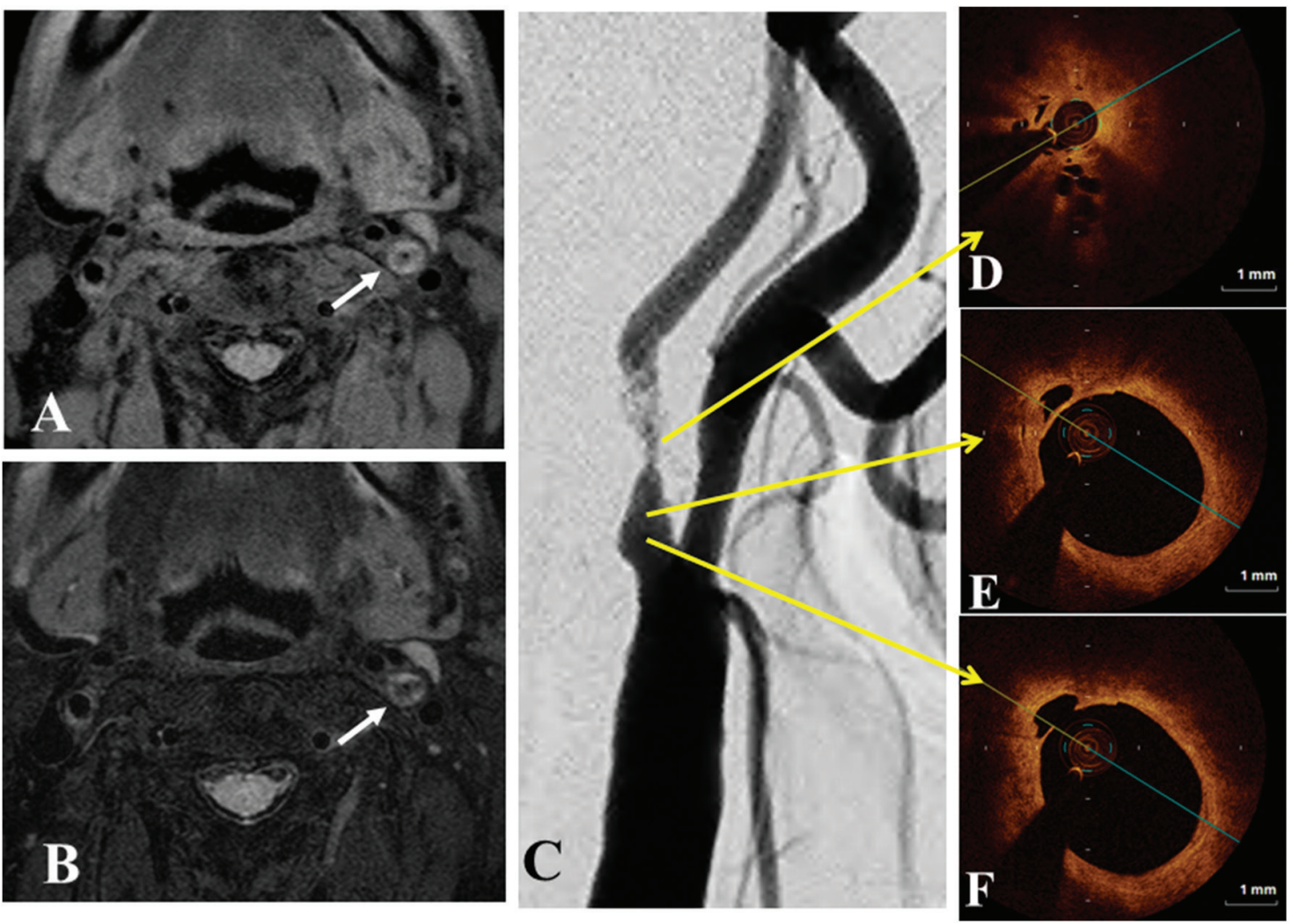

Fig. 1 Magnetic resonance plaque imaging (black-blood method) of the carotid artery (white arrow) showing high intensity signal in part of the carotid plaque (A: T1WI, B: T2WI). Diagnostic digital subtraction angiography revealing severe stenosis of the left carotid artery (C). OCT demonstrating poorly delineated mixed signal-rich area and signal-poor area with some signal-poor defects at the most stenotic site (D), and well-defined homogenous signal-rich thick area with ulceration at another site ( $\mathbf{E}$ and $\mathbf{F})$. OCT: optical coherence tomography; WI: weighted image

asymptomatic severe stenosis of the left cervical internal carotid artery (ICA) according to MRA. During follow-up period after treatment of the minor stoke, a cerebral blood flow (CBF) study using single-photon emission computed tomography (SPECT) demonstrated hemodynamic compromise in the left cerebral hemisphere. Routine carotid ultrasonography revealed remarkable acceleration flow (peak flow velocity: $480 \mathrm{~cm} / \mathrm{s}$ ) and a mixture of hypo- and hyper-echoic signals in the carotid plaque. Magnetic resonance plaque imaging (black-blood method) showed high intensity signal in part of the carotid plaque compared with intensity of the sternocleidomastoid muscle (Figs. 1A and 1B). Over 90\%, severe stenosis (measured by North American Symptomatic Carotid Endarterectomy Trial [NASCET] method) of left ICA was noted by diagnostic cerebral angiography (Fig. 1C).

During cerebral angiography, OCT imaging was performed by the following method.

A 6-Fr guiding catheter was navigated into the left common carotid artery, a 0.014-inch guidewire was advanced carefully across the stenotic lesion, and an OCT imaging catheter (Dragonfly, St. Jude Medical, Inc., St. Paul, MN, USA) was placed at the level of the stenotic lesion. Then, low molecular weight dextran diluted by $50 \%$ in contrast medium was injected through the guiding catheter. The stenotic site was scanned with OCT from the distal section at $20 \mathrm{~mm} / \mathrm{sec}$ using an automatic pull-back device. We used a new-generation OCT imaging system with high-speed resolution and without the balloon-occlusion technique. OCT imaging clearly demonstrated the vascular wall structure.

At the most severe stenotic site (Fig. 1D), OCT imaging showed a homogenous signal-rich area beneath the fibrous cap and an ill-defined homogenous signal-poor area in the deeper layer. Multiple flow void-like signal-poor deficits were also confirmed in the signal-rich area. At another site of the stenosis (Figs. 1E and 1F), OCT imaging demonstrated a well-defined homogenous signal-rich thick layer with ulceration.

Based on these data, the patient was diagnosed with severe stenosis of left ICA with lipid-rich soft plaque with ulceration. He underwent CEA under general anesthesia for carotid artery stenosis. Plaque tissue was removed in 

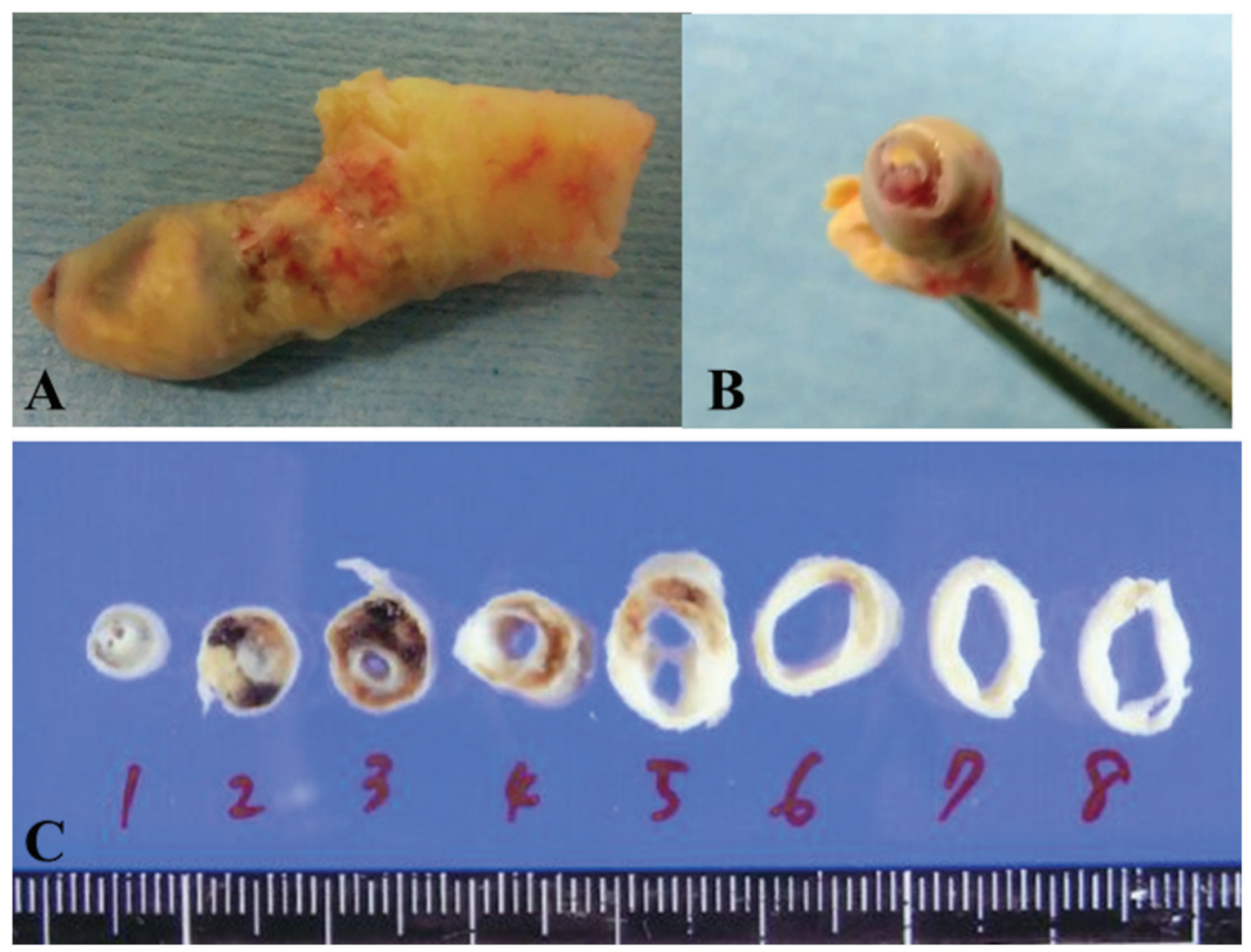

Fig. 2 Carotid plaque mass removed by carotid endarterectomy (A and B). Axis cross sections of carotid plaque after formalin fixation (C).

one mass without any incision during CEA (Figs. 2A and 2B). The excised specimen was fixed with formalin (Fig. 2C), and a pathological section of axis cross section was hematoxylin and eosin stained. Findings for histopathologic assessment were compared with those from preoperative OCT images at the same section levels.

\section{Comparison of OCT findings and pathological findings}

In section 2 of Fig. $\mathbf{2 C}$, which is the most severe stenotic site, the fibrous component around the vascular true lumen was confirmed, and hemorrhage within the plaque or necrotic transformation was confirmed outside of the fibrous component (Fig. 3). The signal-rich region indicated by OCT imaging was shown to be a fibrous component on histopathologic examination. The flow void-like signal-poor round defects on OCT revealed vascular lumens that were present in the thickened fibrous membrane according to histopathologic examination. However, OCT did not visualize the extremely small vascular lumen that was confirmed on histopathologic examination. On histopathologic examination, it was possible to differentiate between plaque lipid component, hemorrhagic transformation, and necrotic transformation. However, it was impossible to differentiate between these findings on OCT, since OCT showed these lesions as homogenous, signalpoor area.

In section 4 of Fig. 2C, the structure of vascular wall (e.g., ulceration and fibrous cap) on OCT image was consistent with that seen on histopathologic examination (Fig. 4).

\section{Discussion}

There was consistency between some components of preoperative OCT characterization of the carotid plaque and pathological findings from CEA specimens. First, vascular wall structure (e.g., ulceration and fibrous cap) was clearly visualized by OCT, and findings from OCT were consistent with those from histopathologic examination. Second, plaque characteristics (e.g., fibrous and soft plaque components, including lipid, necrotic core, and hemorrhagic change) were also consistent between OCT and histopathologic examination. Third, the flow void-like defects on OCT were clearly represented as the vascular lumens on histopathologic examination, and it is likely that neovascularization developed in the plaque with a stable fibrous component.

OCT has been used for plaque characterization during coronary intervention. ${ }^{12-15)}$ Some studies have investigated the 

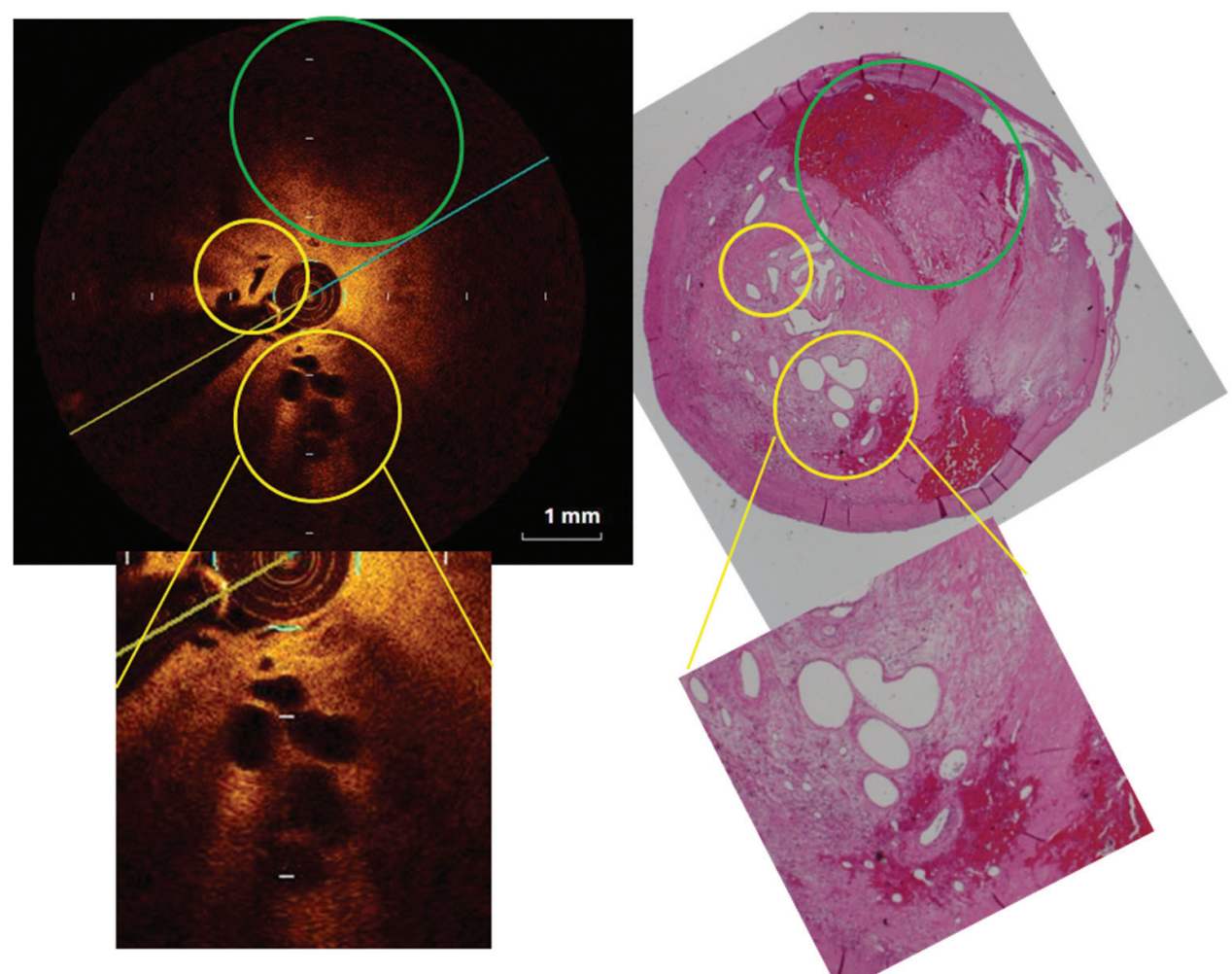

Fig. 3 Comparison of OCT findings (left side) and histopathologic examination of resected specimens (right side) of section 2 of Fig. 2C. Signal-rich area of OCT finding demonstrating fibrous tissue component of pathological specimen. Round signal-poor defects of OCT (yellow circles) consisting of vascular lumens in the fibrous component of pathological specimen (yellow circles). Poor-defined, signal-poor area of OCT (green circle) including both hemorrhagic and necrotic components on histopathologic examination of resected specimen (green circle). OCT: optical coherence tomography

definition of coronary plaque characteristics (fibrous plaque, lipid plaque, and calcified plaque) and the vascular wall formations (fibrous cap, ulceration) by comparing OCT findings and histopathologic examination of resected specimens. ${ }^{16-18}$ According to these reports, OCT shows fibrous plaque as homogenous signal-rich area with a well-defined border, lipid plaque as homogenous signal-poor area with an ill-defined border, and calcified plaque as well-defined, low backscattering, signal-poor area. There have been a few previous studies that compared OCT findings and pathological findings from resected carotid artery plaques., ${ }^{1,2}$ Yoshimura et al. reported the first case of OCT findings of human carotid artery stenosis with soft plaque. ${ }^{1,2)}$ They compared the OCT findings with pathological specimen removed by endarteretomy.

In the present case, comparison of the OCT and pathological findings showed that carotid plaque characteristics were very similar to coronary plaque characteristics. This suggests that the interpretation of coronary artery findings of OCT can be applied to OCT findings of carotid artery plaque.

OCT sometimes demonstrates small round regions with signal-poor, flow void-like findings in a thickened fibrous component in intimal hyperplasia during coronary intervention. ${ }^{16,18,19)}$ These regions are regarded as neovascularization that developed in a thickened and proliferative intima. However, it has not been proven whether the OCT findings of these flow void-like defects are actually vascular lumens. Similar findings are confirmed on OCT findings of the carotid artery, and they are more clearly visualized than those of the coronary artery because the diameter of the carotid artery is larger than that of the coronary artery. If OCT imaging can observe neovascularization structures in detail, it will be helpful to elucidate the mechanism of carotid stenosis, false lumen transformation, and the mechanism of restenosis after CEA or carotid artery stenting. Recently, morphologic features of carotid plaque by OCT have been reported. Shindo et al. observed fibrous cap of carotid plaque by OCT in detail and concluded that a fibrous cap thickness of $<130 \mu \mathrm{m}$ was the threshold for plaque rupture in the carotid artery. ${ }^{11)}$

However, OCT image resolution has some limitations. For example, the lesions visualized as lipid transformations, hemorrhagic transformations, and necrotic 


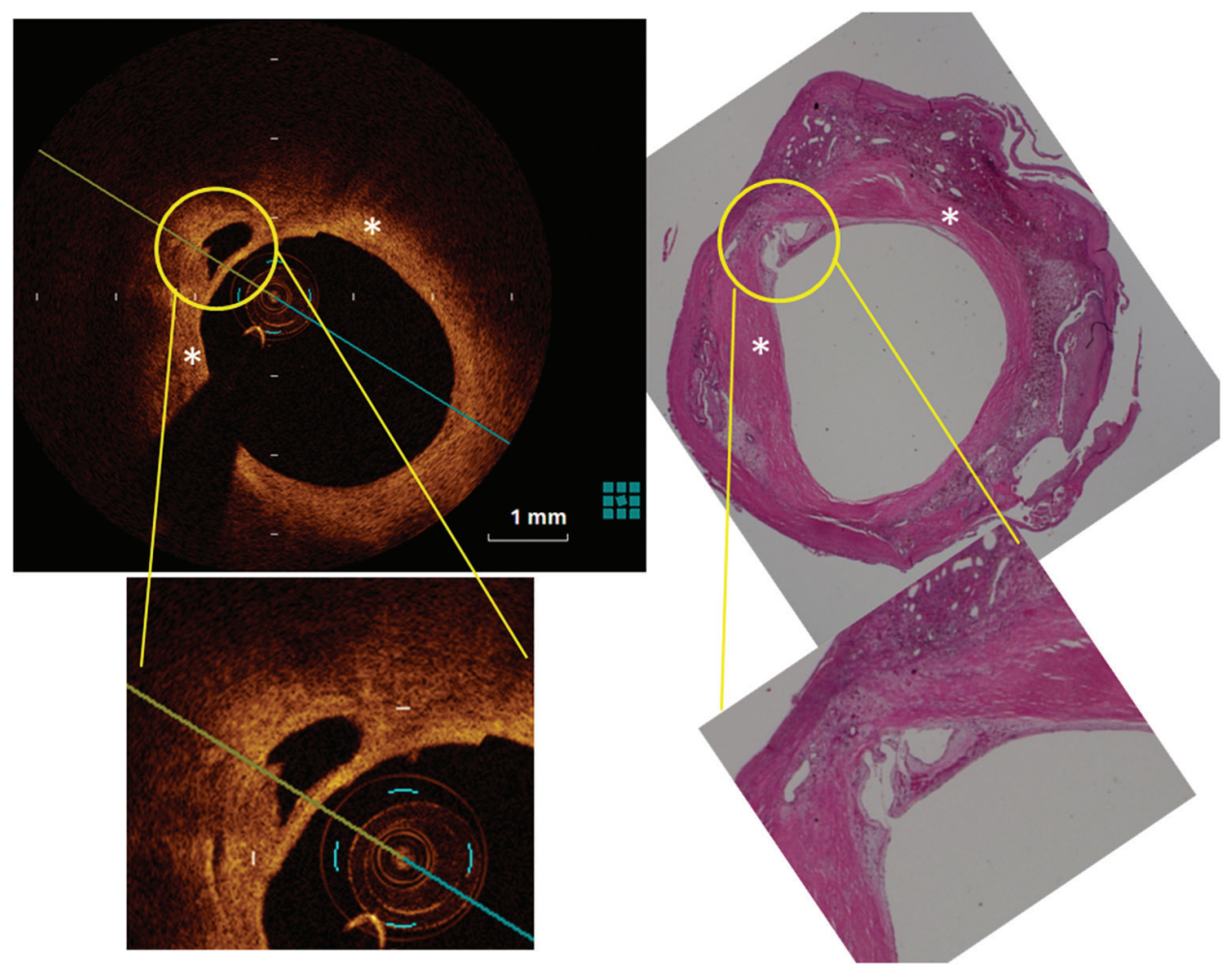

Fig. 4 Comparison of OCT findings (left side) and histopathologic examination of resected specimen (right side) of section 4 of Fig. 2C. Signal-rich layer of OCT finding (asterisk) corresponding to fibrous tissue component of the pathological specimen (asterisk). Signal-poor area with thin signal-rich layer (yellow circle) corresponding to ulceration with fibrous cap of the pathological specimen (yellow circle). OCT: optical coherence tomography

transformations on pathological findings were visualized as a homogeneous, ill-defined, signal-poor area on OCT. Therefore, OCT could not distinguish the difference between these lesions correctly. OCT is more invasive than MRA or 3D computed tomographic angiography and therefore may be relatively limited as a routine preoperative examination. Signal penetration depth of the current OCT system is still low, and it cannot demonstrate the entire diameter of the carotid artery. New generation of OCT imaging is expected and may have improved capabilities.

Although this is only a case report, OCT findings showed a lot of carotid plaque information and vascular wall information. In the future, OCT may be a device allowing detailed observation of carotid plaque morphology in real time. Larger studies are needed to establish the benefits and safety of OCT procedure.

\section{Conclusion}

OCT imaging resolution is very good and its findings accurately represent the pathological findings of carotid plaque.
If it is used properly with understanding of its limitations, it may become one of the useful modalities for evaluating carotid plaque characterization.

\section{Disclosure Statement}

The first author or any of the co-authors have no conflicts of interest to disclose regarding this article.

\section{References}

1) Yoshimura S, Kawasaki M, Hattori A, et al: Demonstration of intraluminal thrombus in the carotid artery by optical coherence tomography: technical case report. Neurosurgery 2010; 67: onsE305; discussion onsE305.

2) Yoshimura S, Kawasaki M, Yamada K, et al: OCT of human carotid arterial plaques. JACC Cardiovasc Imaging 2011; 4: 432-436.

3) Yoshimura S, Kawasaki M, Yamada K, et al: Visualization of internal carotid artery atherosclerotic plaques in symptomatic and asymptomatic patients: a comparison of optical coherence tomography and intravascular ultrasound. AJNR Am J Neuroradiol 2012; 33: 308-313. 
4) Attizzani GF, Jones MR, Given CA, et al: Frequencydomain optical coherence tomography assessment of very late vascular response after carotid stent implantation. J Vasc Surg 2013; 58: 201-204.

5) de Donato G, Setacci F, Sirignano P, et al: Optical coherence tomography after carotid stenting: rate of stent malapposition, plaque prolapse and fibrous cap rupture according to stent design. Eur J Vasc Endovasc Surg 2013; 45: 579-587.

6) Given CA, Attizzani GF, Jones MR, et al: Frequencydomain optical coherence tomography assessment of human carotid atherosclerosis using saline flush for blood clearance without balloon occlusion. AJNR Am J Neuroradiol 2013; 34: 1414-1418.

7) Jones MR, Attizzani GF, Given CA, et al: Intravascular frequency-domain optical coherence tomography assessment of atherosclerosis and stent-vessel interactions in human carotid arteries. AJNR Am J Neuroradiol 2012; 33: 1494-1501.

8) Jones MR, Attizzani GF, Given CA, et al: Intravascular frequency-domain optical coherence tomography assessment of carotid artery disease in symptomatic and asymptomatic patients. JACC Cardiovasc Interv 2014; 7: 674-684.

9) Liu R, Jiang Y, Xiong Y, et al: An optical coherence tomography assessment of stent strut apposition based on the presence of lipid-rich plaque in the carotid artery. $J$ Endovasc Ther 2015; 22: 942-949.

10) Matsumoto $H$, Yako $R$, Masuo $O$, et al: A case of in-stent neoatherosclerosis 10 years after carotid artery stent implantation: observation with optical coherence tomography and plaque histological findings. Neurol Med Chir (Tokyo) 2014; 54: 139-144.
11) Shindo S, Fujii K, Shirakawa M, et al: Morphologic features of carotid plaque rupture assessed by optical coherence tomography. AJNR Am J Neuroradiol 2015; 36: 2140-2146.

12) Ben-Dor I, Mahmoudi M, Pichard AD, et al: Optical coherence tomography: a new imaging modality for plaque characterization and stent implantation. J Interv Cardiol 2011; 24: 184-192.

13) Buja LM: Vascular responses to percutaneous coronary intervention with bare-metal stents and drug-eluting stents: a perspective based on insights from pathological and clinical studies. J Am Coll Cardiol 2011; 57: 1323-1526.

14) Regar E, Ligthart J, Bruining N, et al: The diagnostic value of intracoronary optical coherence tomography. Herz 2011; 36: 417-429.

15) Terashima M, Kaneda H, Suzuki T: The role of optical coherence tomography in coronary intervention. Korean $J$ Intern Med 2012; 27: 1-12.

16) Nakazawa G, Otsuka F, Nakano M, et al: The pathology of neoatherosclerosis in human coronary implants bare-metal and drug-eluting stents. J Am Coll Cardiol 2011; 57: 1314-1322.

17) Nakazawa G: Stent thrombosis of drug eluting stent: pathological perspective. J Cardiol 2011; 58: 84-91.

18) Yabushita H, Bouma BE, Houser SL, et al: Characterization of human atherosclerosis by optical coherence tomography. Circulation 2002; 106: 1640-1645.

19) Takano M, Yamamoto $M$, Inami $S$, et al: Appearance of lipid-laden intima and neovascularization after implantation of bare-metal stents extended late-phase observation by intracoronary optical coherence tomography. J Am Coll Cardiol 2009; 55: 26-32. 